\title{
Primeras experiencias enfermeras en pacientes con mieloma múltiple mediante filtros de alto poro
}

\author{
Víctor Cantín Lahoz, Mercedes Marcén Letosa, Elisa Rojo Zulaica, Blanca Aznar Buil, Ana Vecino Soler
}

Unidad de diálisis, Hospital Clínico Universitario Lozano Blesa, Zaragoza

\section{Introducción:}

El mieloma múltiple es una enfermedad hematológica que produce insuficiencia renal aproximadamente en la mitad de los casos por el efecto nocivo de las cadenas ligeras de inmunoglobulinas. Aparece disfunción renal aguda en el $20 \%$ de los pacientes y requiere tratamiento renal sustitutivo en el $10 \%$ de ellos. Para una respuesta eficaz y revertir el fracaso renal agudo es necesario un diagnóstico y tratamiento precoz de la enfermedad, iniciando el tratamiento quimioterápico y eliminando las cadenas ligeras libres. El uso de membranas de alto poro elimina hasta el $90 \%$ de dichas cadenas permitiendo una mayor recuperación de la función renal. Dicho tratamiento consiste en 6 sesiones de diálisis consecutivas de 6 horas como mínimo que precisa de exhaustivos controles analíticos, monitorización de constantes, administración de fármacos y cuidados del acceso vascular. Este perfil de paciente requiere de un profesional enfermero que ha de estar altamente cualificado en el manejo del procedimiento técnico y en el acompañamiento terapéutico. En nuestra unidad hemos realizado el tratamiento a ocho pacientes diagnosticados de mieloma múltiple con fracaso renal agudo entre julio 2011 y marzo 2013. Hemos sido el centro de referencia en Aragón para realizar este tratamiento por disponer de hematólogo y nefrólogo de guardia además de disponer de agua ultra pura, que es condición indispensable para realizar esta técnica. Todos los pacientes al inicio eran portadores de catéter temporal yugular. Se realizaron un total de 95 sesiones, en las cuales las complicaciones e intervenciones más frecuentes fueron: mal funcionamiento del catéter, coagulación del circuito y del dializador, hipotensiones, administración de tratamiento antibiótico por infección de catéter, transfusiones de concentrados de hematíes y cambio del acceso vascular. Algunos pacientes precisaron de tratamiento ansiolítico durante el proceso. La técnica fue efectiva en cuanto a disminución de cifras de creatinina y de cadenas ligeras libres en suero. Se finalizó el tratamiento cuando éstas estuvieron por debajo de $500 \mathrm{mg} / \mathrm{L}$. De los ocho pacientes, seis recuperaron la función renal y dos resultaron diálisis-dependientes. Tres de ellos fallecieron a consecuencia de la evolución desfavorable de su enfermedad hematológica. El tratamiento ha conseguido reducir las cadenas ligeras, lo que demuestra la eficacia del dializador de alto poro. Los casos de no recuperación de función renal tienen relación con la demora del diagnóstico (que conlleva una instauración tardía del tratamiento) y con un tratamiento quimioterápico ineficaz. Los registros enfermeros que actualmente manejamos en hemodiálisis convencional son insuficientes para reflejar correctamente estas sesiones de mayor duración y complejidad de cuidados. Por lo cual, hemos elaborado una nueva gráfica más completa, en la que podemos recoger adecuadamente los datos necesarios para establecer unos cuidados que confieran seguridad y continuidad.

\section{Referencias Bibliográficas}

1. Irish $A B$, Winearls $C G$, Littlewood T. Presentation and survival of patients with severe renal failure and myeloma. Q J Med 1997;90:773-780.

2. Montseny JJ, Kleinkecht $D$, Meyrier $A$, Vanhille $P$, Simon P, Purna A et al. Long-term outcome according to renal histological lesions in 118 patients with monoclonal gammopathies. Nephrol Dial Trasplant 1998;13:1438-45.

3. Kyle RA, Gertz MA, Witzig TE, Lust JA, Lacy $M Q$, Dispenzieri A, et al. Review of 1027 patients with newly diagnoses multiple myeloma. Mayo Clin Proc 2003;78:21-33. 
4. Martín Reyes $G$, Valera $A$, Frutos MA, Ramos $B$, Ordóñez V, López de Novales E. Supervivencia de pacientes con mieloma tratados con diálisis. Nefrología 2003;XXIII(2):131-136.

5. Hutchison CA, Plant T, Drayson M, Cockwell $P$, Kountouri M, Basnayake $K$ et al. Serum free light chain measurement aids the diagnosis of myeloma in patients with severe renal failure. BMC Nephrology 2008;9(11).

6. Hutchison $C A$, Cockwell $P$, Stringer $S$, Bradwell A, Cook M, Gertz MA, et al. Early reduction of serum-free light chains associates with renal recovery in myeloma multiple. J Am Soc Nephrol 2011;22:1129-36.

7. Zucchelli $P$, Pasquali $S$, Cagnoli $L$ et al. Controlled plasma exchange trial in acute renal failure due to multiple myeloma. Kydney Int 1988;33:1175-1180.

8. Johnson WJ, Kyle RA, Pineda AA, O'Brien PC Holley PC. Treatment of renal failure associated with multiple myeloma. Plasmapheresis, hemodialysis and chemotherapy. Arch Interm Med 1990;150:863-9.

9. Clark WF, Stewart AK, Roca GA, Sternbach M, DM Sutton, Barrett BJ et al. Plasma exchange when myeloma presents as acute renal failure. Ann Intern Med 2005;143:773-780.

10. Leung N, Gertz MA, Zeldenrust SR, Rajkumar SV, Dispenzieri A, Fervenza FC et al. Improvement of cast nephropathy with plasma exchange depends on the diagnosis and reduction of serum free light chains. Kidney Int 2008;73:1282-1288.

11. Levy F, Camarero V, Torres G, Hijazi B. Plasmaféresis como tratamiento adyuvante en el fallo renal agudo secundario al riñón de mieloma. Nefrología 2009;29(3):283-284.

12. Hutchison CA, Bradwell AR, Cook M, Basnayake $K$, Basu S, Harding $S$ et al. Treatment of acute renal failure secondary to multiple myeloma with chemotherapy and extended high cut-off hemodialysis. Clin J Am Soc Nephrol 2009;4:745-754.

13. Shum HP, Chan KC, Chow CC, Kho B, Yan WW. Cast nephropathy with acute renal failure treated with high cut-off haemodialysis in a patient with multiple myeloma. Hong Kong Med J 2010;16(6):489-492.
14. Mallol A, Folch MJ, Renau E, Cerrillo V. Hemodiálisis con dializadores de poro grueso para tratar el fracaso renal agudo del paciente con mieloma. Rev Soc Esp Enferm Nefrol 2011;14(2).

15. Álvarez R, Martín F, Abascal JA, Berni A, Dourdil $\mathrm{V}$, Palomera L. Tratamiento de la IRA secundaria a mieloma múltiple con filtro de high cut-off. Dial Traspl. 2013;34(1):36-40.

16. Kleeberg L, Morgera S, Jakob C, Hocher B, Schneider $\mathrm{M}$, Peters $\mathrm{H}$ et al. Novel renal replacement strategies for the elimination of serum free light chains in patients with kappa light chain nephropathy. Eur J Med Res. 2009;14(2)47-54.

17. Heyne N, Denecke B, Guthoff M, Oehrlein K, Kanz L. Extracorporeal light chain elimination: high cut-off (HCO) hemodialysis parallel to chemotherapy allows for a high proportion of renal recovery in multiple myeloma patients with dialysis-dependent acute kidney injury. Ann Hematol 2012;91:729-735.

18. Khalafallah A, Wuong $S$, Amor $S$, Mohamed $M$, Mace R, Khalil R et al. Early aopplication of high cutt-off haemofialysis for de-novo myeloma nephropathy is associated with long-term dialysis-independency and renal recovery. Mediterr Hematol J Infect Dis. 2013;5(1):

19. Echabe L, Iturralde I, González E, Telleria A, Rodrigo T, Rivas M. Hemodiálisis con filtro de alta permeabilidad para eliminación de cadenas ligeras en pacientes con mieloma. Disponible en: http:// www.revistaseden.org/files/2736_23\%20\%20 1620.pdf con acceso 24 abril 2013.

20. Martin Reyes $G$, Toledo R, Torres A, Sola E, Blanca $L$, Fuentes $L$ et al. Tratamiento con hemodiálisis del fracaso renal agudo en el mieloma múltiple con filtros de alto poro (high cut-off). Nefrol 2012;32(1):35-43.

21. Hutchison CA, Cook M, Heyne N, Wiesel K, BiIlingham $L$, Bradwell $A$ et al. European trial of free light chain removal by extended haemodialysis in cast nephropathy (EuLITE): A randomized control trial. Trials 2008;9:55.

22. Grima DT, Airia $P, A t t a r d ~ C$, Hutchison CA. Modelled cost-effectiveness of high cut-off hemodialysis compared to standard haemodialysis in the management of myeloma kidney. CMRO 2011;27(2):383-391. 